mittels. (Korrespondenz 6; Chem. Zentralbl. 1915, II, 1317.) - Die Gegenwart eines Konservierungsmittels im Eigelb wurde in der Weise festgestellt, daß bei Impfversuchen mit Hefe usw. keine Gärung erfolgte. Der chemische Nachweis eines Konservierungsmittels kann durch diese biologische Methode unterstützt werden.

P. Neumann.

\title{
Patente.
}

Dr. Georg Fendler in Berlin.Schöneberg: Verfahren zur Reinigung des durch Behandiung von Eigelb mit Aceton erhaltenen Lecithinpräparates. D.R.P. 372057 vom 15. Februar 191s (Patentbl. 1914, 35, 877). - Man durchfeuchtet das Lecithinpräparat mit Wasser oder Milch und trocknet bei gewöhnlicher oder böherer Temperatur.

Dr. H. Martin in Wien: Herstelluog von Lecithinpräparaten aus Eigelb. D.R.P. 286907 vom 11. November 1913 (Patentb]. 1915, 36, 1360). - Das Eigelb wird ohne vorgängige Entfernung des Kieröles und sunstiger Nebenbestandteile unmittelbar mit einem Spaltungsmittel für Lecithineiweikverbindungen, insbesondere mit heißem Alkohol behandelt und das Gemisch nach der Entfernung des Spaltungsmittels im Gemenge mit körpergebenden Stoffen getrocknet.

Erben des Karl Alfred Fischer in Berlin: Verfahren zur Herstellung hochprozentiger Lösungen von Lecithin jn Ôlen und Fetten. D.R.P. 286061 vom 2. Februar 1913 (Patentbl. 1915, 36, 1139). - Man nimmt von einem Gemisch von 75 Teilen Olivenöl und 25 Teilen Ölsänre 60 Teile und setzt dazu 80 Teile einer $50 \%$-igen alkobolischen Lecithinlösnng, die durch fraktionierte Küblung zwecks Entfernung der schwer löslichen Anteile des lecithins erhalten wurde. Man giefst das Gemisch auf eine flache Schale und erwärmt es auf $50-60^{\circ}$, um den Alkohol zu verjagen. Das Produkt enthält fertig $40 \%$ Lecithin. Es hält sich auch am Lichte; selbst nach längerem Stehen fällt kein Lecithin wieder aus.

Hugo Kraft in Köln a. Rh.: Verfahren zur Darstellung von Jodeisene lecithin. D.R.P. 292961 vom 4. September 1913 (Patentbl. 1916, 37, 722). - Das Verfahren ist dadurch gekennzeichnet, daß man gesättigte alkoholische Lösungen von Lecithin mit alkoholischen Lösungen berechneter Mengen von Eisenchlorid und Jod in der Wärme, vorzugs. weise bei $60^{\circ} \mathrm{C}$, behandelt. Das nach vorliegendem Verfahren erhaltene Jodeisenlecithin, welches Phosphor, Eisen, Jod und Stickstoff organisch gebunden enthält, stellt eine Verbindung von therapeutischem Werte dar, welche insbesondere den Vorteil bietet, dak das Jod infolgseiner organisehen Binduag den Magen nicht belf̈stigt, während es vom Darm leicht resorbiert wird. M. Sehütz.

\section{Wurzelgewächse, Gemüse und sonstige pflanzliche Nahrungsmittel.}

Ferlinand Kryz: Eine Methode zur raschen Ermittelung des. spezifischen Gewichtes für die technische Kartoffelprüfung. (Zeitschr. f. d. landw. Versuchsw. in Deutschösterreich 1919, 22, 127-130.) - Nach dieser neuen, sog. Bürettenmethode verfährt man folgendermaßen: Mit Hilfe einer Messingröhre (Korkbohrer) von 8-10 mm Durchmesser stanzt man einen Zylinder aus der zu untersuchenden Kartoffel aus, bringt ihn sofort in ein tariertes Schälchen und ermittelt auf der analytischen Wage rasch sein Gewicht. Darauf wird sein Volumen derart bestimmt, daf man zunächst in eine Bürette soviel destilliertes Wasser einfüllt, daf der Meniskus mit einem in der unteren Bürettenhälfte gelegenen Kubikzentimeterteilstrich übereinstimmt, und sodann das gewogene Stanzstüek hinuntergleiten und luftblasenfrei untersinken läßrt. Der Niveauuntersehied des Wasserspiegels ist das Volumen des Zylinders und der Quotient aus dem Gewicht und dem Volumen das spezifische Gewicht. Zweckmäßig stanzt man aus einer größeren Anzabl Kartoffeln der zu untersuchenden Probe je einen Zylinder heraus, die man gemeinsam in einer tarierten Schale wägt und dann hintereinander in eine weniger als halb gefüllte Bürette bringt, worauf man wie oben verfährt. Da das Volumen eines Stanzzylinders von $8 \mathrm{~mm}$ Durchmesser und $40 \mathrm{~mm}$ Länge genau $2 \mathrm{~cm}$ beträgt, findet nach Eỉnbringen von 10 Stanzstücken in die Bürette eine Erhöhung des Wasserspiegels um etwa 20 bis $25 \mathrm{ccm}$ statt. Diese Methode liefert Ergebnisse, die der pyknometrischen viel näher kommen als der Stohmann'schen Methode.

E. Dinslage. 
Otto Matzdorf und Walter Kühne: Uber die Fettbestimmung in Trockenkart offeln. (Zeitschr. gesamt. Getreidew. 1919, 11, 44-45). - Da Verff. vermuteten, daß auch das Fett in den Kartoffeltrockenprodukten sich ebenso oder zumindest ähnlich verhalten müsse wie in Backware, beschlossen sie eine Reihe von Kartoffelpräparaten gewichtsmäßig auf Fett zu prüfen, jedesmal sowohl direkt mittels Âtherextraktion als auch nach erfolgter Hydrolyse. Bezüglich der letzteren machten sie sich die Feststellungen von Neumann, Kalning und Grujic bei der Prüfung der Verfahren von Weibull, Polenske und Berntrop zu eigen und benutzten das von den Verfassern abgeänderte Verfahren (Z. 1914, 27, 748). Die Ergebnisse sind in einer Tabelle zusammengestellt. Wenn auch in einigen Fällen nach der Hydrolyse niedrigere Werte gefunden wurden, so bestätigt die Tabelle immerhin, daß der Grundgedanke über die modifizierte Fettbestimmung in Backware durchaus gerechtfertigt war.

P. Neumann.

H. L. Huenink und Ldward Bartow: Der EinfluB des Mineralge. halts des Wassers a $\mathrm{K}$ onserven. (Journ. Ind. and Engin. Chem. 1915, 7, 495-496.) - Die Verff. berichten über Kochversuche von Bohnen mit Wasser, dem die verschiedensten Aschenbestandreile zugesetzt waren. Es ergab sich, daß es für die Beschaffenheit der fertigen Konserven von großem Einflusse ist, ob hartes oder weiches Kochwasser verwandt wurde. Die Versuche sollen weiter ausgedehnt werden.

C. Grimme.

W. Bouhon: Gesundheitsschädlicher Rhabarber. (Bericht der Städt. Lebensmittel-Untersuchungsanstalt Altenburg 1916, 483.) - Nach Genuß von Büchsenrhabarber waren etwa 100 Mann eines Ersatzbataillons an Durchfall, Erbrechen, Mattigkeits- und Schwindelgefühl erkrankt. Daß die Erkrankungen auf den Genuß des Büchsenrhabarbers zurückzuführen waren, stellte der Unterarzt des Bataillons dadurch fest, daß er selbst $1 / 2$ Pfund der Konserve genoB und danach unter den gleichen Erscheinungen erkrankte. Von dem Vorrat wurden daraufhin Proben entnommen, die auffallend große Mengen $O x$ alsäure und $\mathrm{Z}$ inn bis $z u \quad 0,04 \%$ enthielten. Auf Grund ärztlichen Gutachtens wurde der noch vorhandene Vorrat eingezogen und vernichtet.

C. Mai.

A. Loewy und v. d. Heide: Uber die Verdaulichkeit der Pilze. (Verhandl. d. Physiol. Gesellschaft zu Berlin 1915, 40, II, 19-24; Chem. Zentralbl. 1916. I, 621.) - Pilze sind in der Form, in der sie gewöhnlich genossen werden, trotz des reichlichen Gehaltes an organischen und besonders an stickstoffhaltigen Bestandteilen ein verhältnismäßig wenig wertvolles Nahrungsmittel, weil nach allgemeiner Annahme ihr Nährstoffinhalt schlecht ausgenutzt wird. Die Verff. präften in Ausnutzungsversuchen an Steinpilzen, ob durch feine Vermahlung die Ausnutzbarkeit des Sticketoffes verbessert werden könnte. Dies ist nur in ganz geringem Maße der Fall. Wenn man von dem Stickstoff der Pilze die auch bei künstlicher Verdaunng unverdauliche Menge (Chitin und äbnliche Verbindungen) in Abzug bringt, so zeigen die Versuche, daß die resorbierbare Menge gar nicht schlecht ausgenutzt wird. Die Ausnutzung dieses Anteils betrug bei in gewöhnlicher Weise zerkleinerten Pilzen 81, bei feingemahlenen $85 \%$.

Max Muiller.

E. Hermann: Behandlung und Untersuchung der Trockenpilze. (Pharm. Zentralh. 1919, 60, 5-7 und $21-25$.)

H. Serger: Zur Fabrikation, Wertbestimmung und Verwertang von Dörrgemüse. (Konserven-Industrie 1916, 7/10; Chem. Zentralbl. 1916, II, 940.) Verfasser beschreibt einige Aulagen zur Herstellung von Dörrgemüse. 


\section{Patente.}

Verein der Spiritusfabrikanten in Dentschland in Berlin: Verfahren zur Konservierung von Kart offeln. D R.P. 286106 vom 30. Mai 1913 (Patentbl. 1915, 36, 1150). - Die Kartotfeln werden im rohen Zustand gerieben und in wasserdichten Gruben mit Milchsäurepilzreinkultur geimptt (ev. Zusatz von Zuckerrübenschnitzel oder -Melasse).

Verein der Spiritusfabrikanten in Dentschland in Berlin: Verfahren der Einsäuerung von rohen Kartoffeln. D.R.P. 287487 vom 13. Februar 1914 (Patentbl. 1915, 36, 1448). - Man setzt den Kartoffeln bei einer Zerkleinerung, die keine Saftabscheidung hervorbringt, Kochsalz zwecks Saftabscheidung hinzu und impft darauf die Masse mit Reinkulturen von Bakterien.

R. Kleinbrod gen. Klein in Straß̧burg i. E.: Verfabren zur Herstellung eines Nahrungs- und Genufmittels aus Johannisbrot. D.R.P, 285153 vom 12. Oktober 1913 (Patentbl. 1915, 36, 1407). - Johannisbrot wird der Einwirkung von Dampf unter Druck und ständiger Bewegung der Masso ausgesetzt, worauf die Masse getrocknet und mit Geschmacksstoffen nach Zerkleinerung versetzt wird.

M. Schiitz.

\section{Gebrauchsgegenstände.}

\section{Technische Fette und Öle, Seifen, Haxze, Wachse.}

Carl Lonis Sehumann: Polymerisation von chinesischem Holzöl. (Journ. Ind. and Engin. Chem. 1916, 8, 5-15.) - Das vom Verf. zu seinen Versuchen benutzte Holzöl hatte nachstehende Kennzahlen: Säurezahl 4,7, Verseifungszahl 190,9, Jodzahl (W ijs) 167,7. Brechungsindex $\left(20^{0}\right)=1,5182$, Spez. Gewicht $\left(15,5^{0}\right) 0,9412$, Fettsäuren $95,1 \%$, Oxysäuren $1,7 \%$. Die für Holzöl charakteristische Eläomargarinsäure wurde, wie folgt, isoliert: $5 \mathrm{~g}$ Ol werden in üblicher Weise verseift und die Gesamtfettsäuren isoliert. Letztere werden in $40 \mathrm{ccm}$ absolutem Alkohol gelöst, die Lösung wird auf $0^{\circ}$ abgekühlt, dann gibt man bis zur beginnenden Trübung Eiswasser $(14-14,5 \mathrm{~cm})$ hinzu, bringt den Niederschlag durch Erwärmen auf $5^{\circ}$ wieder in Lösung und läbt über Nacht im Eisschrank stehen. Hierbei fällt die Eläomargarinsäure in rhombischen Tafeln aus. Abfiltrieren im Eistrichter, das Filtrat unter vermindertem Druck im Wasserbade stark konzentrieren, dureh abermaliges Destillieren mit absolutem Alkohol die zurückbleibenden Fettsäuren entwässern, Im Kohlensäurestrome trocknen und wägen. Differenz zwischen Einwage und Rückstand = Eläomargarinsäure, wobei für im Filtrat gelöst gebliebene Eläomargarinsäure eine Korrektion von $0,132 \mathrm{~g}$ angebracht werden muf. Bei der Bestimmung in polymerisiertem Holzöle kann der Gehalt unter $0,132 \mathrm{~g}$ für $5 \mathrm{~g}$ Öl sinken, sodaß die Ausbeute $=0$ ist. In diesem Falle gibt man bei einer erneuten Bestimmung $0,5 \mathrm{~g}$ frisches, unpolymerisiertes $O \mathrm{Ol} \mathrm{hinzu,} \mathrm{dessen} \mathrm{Gehalt} \mathrm{an} \mathrm{Eläomargarinsäure} \mathrm{in} \mathrm{einem}$ Sonderversuche bestimmt wird. - In bezug auf die Vorgänge bei der Polymerisation von Holzöl konnte Verf. feststellen, daß sich zunächst bei der Gelatinierung durch Erhitzen an der Luft merkliche Mengen von Oxydationsprodukten bilden, welche als positive Katalysatoren auf die Gelatinierung wirken. Alleiniges Erhitzen auf $150^{\circ}$ unter Einblasen von Luft bewirkte in $21 / 2$ Stunden vollkommenes Festwerden, Erhöhung der Temperatur auf $200^{\circ}$ drückt die Gelatinierungsdauer auf unter 1 Stunde herab, während ein Erhitzen in offener Schale ohne Lufteinblasen bei $150^{\circ}$ erst in 30 Stunden zur Gelatinierung führte. Setzt man dem Öle polymerisiertes Öl zu, so wirkt dieses als Katalysator und beschleunigt in allen Fällen erheblich die Gelatinierung, selbst in inerten Gasen, also unter Ausschlub von Sauerstoff. Zusätze von reduzierenden Metallen verlangsamen die Gelatinierung, können sie aber nicht verhindern. 'Die angewandten Temperaturen müssen jedoch erhöht werden. - Die Vorgänge bei der Gelatinierung von Holzöl verlaufen so, daf sich zunächst infolge Auflösung der Hälfte der Doppelbindungen des Eläomargarinsäuretriglycerids ein dimolekulares Triglycerid bildet, welches nach Erreichung einer bestimmten Menge infolge seiner Un- 